Revista de Matemática: Teoría y Aplicaciones 2010 17(1) : 69-80

CIMPA - UCR ISSN: 1409-2433

\title{
DISEÑO ÓPTIMO DE REDES Y ENRUTAMIENTO DE COMPUTADORAS: CASO PRÁCTICO
}

\author{
OPTIMAL DESIGN OF NETWORKS AND \\ ROUTING OF COMPUTERS: PRACTICAL CASE
}

\author{
Carlos A. PÉrez Enríquez * \\ Sergio G. De-LOS-Cobos-Silva**
}

Received: 16 Apr 2009; Revised: 27 Oct 2009; Accepted: 30 Oct 2009

Palabras clave: Optimización de redes, Problema tipo Steiner, Algoritmos heursticos.

Keywords: Networks optimization, Steiner problems, heuristics algorithms.

Mathematics Subject Classification: 8M10, 90C35, 65K99.

*Departamento de Ingeniería Eléctrica, Universidad Autonónoma Metropolitana Iztapalapa, Av. Michoacán y La Purísima s/n, Col. Vicentina, Del. Iztapalapa, México D.F., C.P. 09340 México; E-Mail: carlosperez@mythosmexico.com

***Misma dirección que C.A. Pérez. E-Mail: cobos@xanum.uam.mx 


\title{
Resumen
}

En este trabajo se introduce al problema de enrutamiento y diseño óptimo de redes de computadoras, las cuales deben de satisfacer ciertas condiciones prácticas de interconectividad. Se presenta aproximación por medio de los problemas tipo Steiner y se realiza la búsqueda de soluciones robustas y económicas.

\begin{abstract}
This work introduced to the problem of routering and optimum design of networks of computers that must satisfy certain practical conditions of interconnectivity. This paper show an approach style Steiner problems, and search robust and more economic solutions.
\end{abstract}

\section{Introducción}

Una red de comunicaciones se compone de un conjunto de nodos emisoresreceptores de información, conectados por enlaces que permiten la transmisión de la información de modo unidireccional, bidireccional o multidireccional. Habitualmente se suele diferenciar entre ciertos nodos, denominados unos como nodos terminales de la red de comunicaciones, sobre los que se plantean requisitos de conectividad, y otros nodos que se utilizan exclusivamente como conectores intermedios en los caminos de comunicación de información entre nodos terminales. El rápido desarrollo de la infraestructura de redes de comunicaciones, diseño de software y servicios por Internet ha sido propulsado por la gran demanda de comunicaciones de datos en los últimos años. Por este motivo, se ha renovado el interés en los problemas de diseño estructural a que se refiere el ruteo de información.

Una de las principales metas en la actualidad, es lo que se conoce como el diseño de una red confiable. Usualmente la minimización de los costos de las conexiones en una red de comunicaciones y la maximización de su confiabilidad respecto a la integridad y confianza de la información enviada y recibida parecerían que son objetivos contrapuestos. La confiabilidad la consideraremos en este trabajo como los requerimientos de conectividad (número de posibles alternativas de conexión entre nodos y se considerará como un parámetro dado por el usuario) entre equipos de cómputo, es decir el número mínimo de caminos distintos entre nodos, proporcionando a su vez buenas velocidades de conexión. Como ejemplo, un modelo que minimice el costo total de la red sin contemplar restricciones adicionales de conectividad (como los introduce la exigencia de que exista 


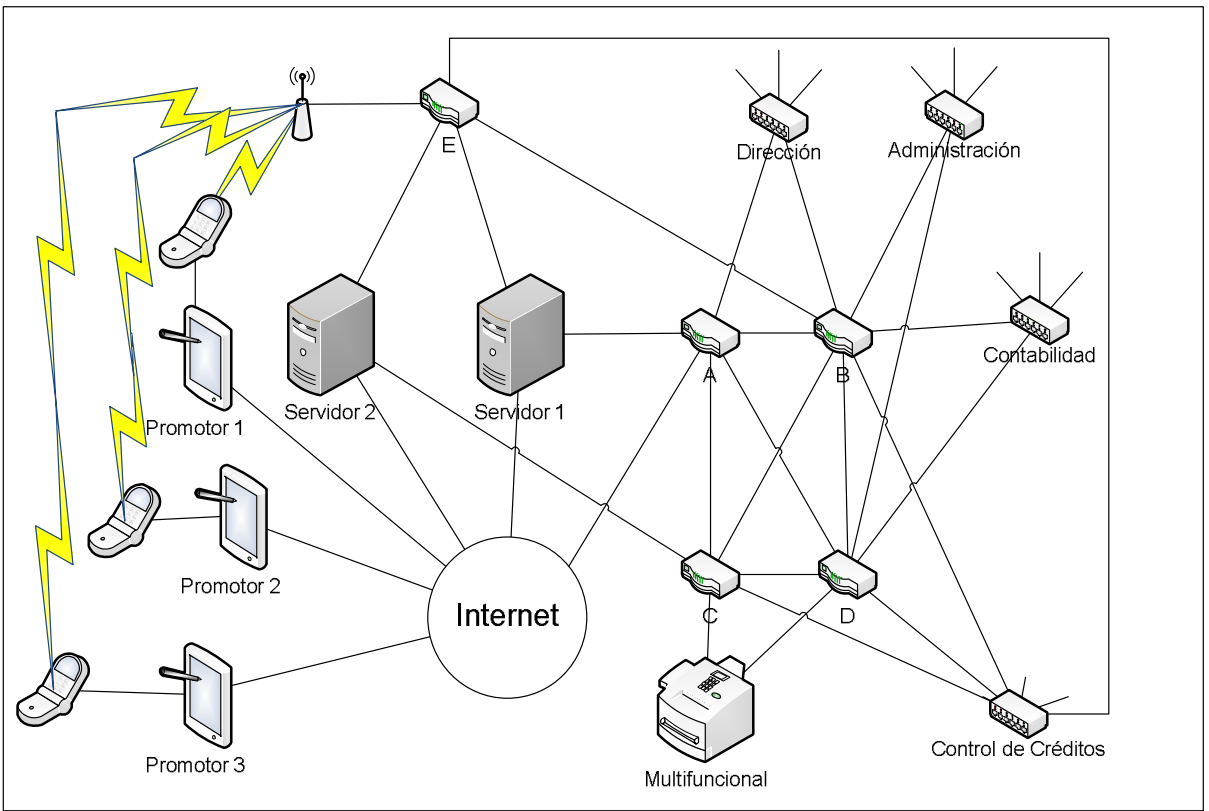

Figura 1: esquema de conexiones de la empresa Alfa.

un mínimo número de caminos de conectividad entre pares de nodos terminales) conduciría a una topología de red en forma de árbol. Esta clase de soluciones, que no introducen redundancia de caminos, no son útiles en la mayoría de los escenarios de la vida real, ya que no son capaces de tolerar ni siquiera una simple falla en uno de los componentes de la red. Cualquier deficiencia en uno de los nodos o en algún enlace afectaría la operatividad de la red, impidiendo la comunicación al menos entre un par de nodos terminales.

\section{Definición del problema}

A continuación se presenta un problema práctico de cierta empresa que en adelante la denominaremos como la compañía Alfa, esta empresa cuyo giro es el financiamiento para remodelación de viviendas tiene como una de sus bases el empleo adecuado de tecnología para lograr sus propósitos. La organización interna de la empresa Alfa contiene los siguientes departamentos: 
1. Dirección: Este departamento está encargado de la coordinación de todo el personal que labora en la empresa. Debido a su poder de toma de decisiones que afectan el desempeño global de la compañía, este departamento debe poseer acceso a la información y comunicación con su personal de manera constante e ininterrumpida.

2. Control de Créditos: Tiene bajo su responsabilidad la autorización o denegación de los créditos gestionados por los promotores a solicitud de los clientes. Como los préstamos son el principal vehículo financiero de la compañia, se requiere que el personal de este departamento tenga acceso a los sistemas de autorización de manera inmediata.

3. Contabilidad: Sección encargada de los registros contables de ingresos y egresos. El retraso temporal de sus funciones en caso de falla con el sistema central de información no supone un alto riesgo en la salud financiera de la compañía.

4. Administración: Se encarga de controlar, planear y dirigir las operaciones básicas de mercadotecnia, recursos financieros y recursos humanos. El acceso al sistema central informático no es necesario de manera inmediata.

5. Promotores: Esta área tiene la responsabilidad de establecer el contacto con los clientes. Este departamento presenta uno de los mayores retos tecnológicos ya que los promotores son agentes de campo y requieren tener la capacidad de conexión remota de manera inmediata, al sistema central de información a través de los equipos portátiles.

Todos estos departamentos usarán un sistema informático central capaz de integrar sinérgicamente la información proporcionando la confiabilidad y eficiencia necesaria para otorgar una ventaja competitiva sobre compañías similares.

El sistema informático central estará instalado en al menos dos equipos de cómputo, ubicados en diferentes lugares mejorando así la capacidad de operación del sistema para que durante eventualidades no previstas no se vea afectada. Se presenta un esquema de conexiones de la empresa alfa en la Figura 1, asimismo el Cuadro 1 se indican los costos mensuales en unidades monetarias(u.m.) en que se incurre por cada conexión entre nodos, así como las velocidades de enlace que proporcionan. En el 
Cuadro 2 se puede observar los requerimientos mínimos de conectividad entre los equipos de la empresa Alfa, estos requerimientos son parámetros suministrados por el usuario (la empresa Alfa en nuestro caso).

Las claves utilizadas en el Cuadro 2 son:

1. Operación no crítica que no requiere redundancia.

2. Toma de decisiones del sistema principal.

3. Toma de decisiones sistema de respaldo.

4. Sincronización de servidores.

5. Acceso remoto.

\section{$3 \quad$ El problema Steiner generalizado (PSG)}

El problema Steiner generalizado es NP-difícil, tal como se indica en el compendio de problemas de Kahn y Crescenzi(2003), tanto en su versión con requerimientos de caminos disjuntos en aristas como en su versión para caminos disjuntos en nodos. Los problemas de $k$ conexión, donde todos los nodos terminales tienen los requerimientos de $k$ caminos disjuntos, son asimismo NP-difíciles. El propio problema del árbol de Steiner, que implica un solo camino para cada par de nodos terminales, planteando la restricción menos general en cuanto al número de caminos exigidos, es NP-completo(Crescenzi y Kann(2005)).

A continuación se presenta una formulación matemática general que puede utilizarse como base para la solución del problema, y se presentan variantes que simplifican la especificación general del problema y antecedentes de aplicación de técnicas heurísticas para la resolución del problema generalizado y sus variantes. Se presenta una formulación del problema Steiner generalizado basada en la incluida en el compendio de Kahn y Crescenzi(2005) de problemas de optimización para los cuales no existe algoritmo conocido de resolución eficiente en tiempo polinomial, es decir que termine con solución óptima global en tiempo polinomial. Cabe mencionar que puede existir ocasiones en que ni siquiera exista una solución factible, por lo que se tendrá que relajarse las condiciones, y aún así tampoco habría necesariamente una solución óptima y se tendría que recurrir a la idea de soluciones óptimas locales o de soluciones de tipo multiobjetivo o de soluciones de tipo de dominancia.

Considérense los siguientes elementos: 


\begin{tabular}{cc|cc}
\hline \hline Nodo 1 & Nodo 2 & Costo mensual & $\begin{array}{c}\text { Velocidad } \\
\text { de enlace (kbps) }\end{array}$ \\
\hline Dirección & $\mathrm{A}$ & 50.00 & 100000 \\
Dirección & $\mathrm{B}$ & 50.00 & 100000 \\
Administración & $\mathrm{B}$ & 50.00 & 100000 \\
Administración & $\mathrm{D}$ & 50.00 & 100000 \\
Contabilidad & $\mathrm{B}$ & 50.00 & 100000 \\
Contabilidad & $\mathrm{D}$ & 50.00 & 100000 \\
Control de Créditos & $\mathrm{C}$ & 50.00 & 100000 \\
Control de Créditos & $\mathrm{D}$ & 50.00 & 100000 \\
Control de Créditos & $\mathrm{B}$ & 50.00 & 100000 \\
Control de Créditos & $\mathrm{E}$ & 50.00 & 100000 \\
Servidor 1 & $\mathrm{A}$ & 50.00 & 100000 \\
Servidor 1 & $\mathrm{C}$ & 50.00 & 100000 \\
Servidor 1 & $\mathrm{E}$ & 50.00 & 100000 \\
Servidor 1 & Internet & 950.00 & 2000 \\
Servidor 2 & $\mathrm{C}$ & 5000.00 & 10000 \\
Servidor 2 & $\mathrm{E}$ & 5000.00 & 10000 \\
Servidor 2 & Internet & 950.00 & 2000 \\
Promotor 1 & Internet & 200.00 & 50 \\
Promotor 1 & $\mathrm{E}$ & 2600.00 & 500 \\
Promotor 2 & Internet & 200.00 & 50 \\
Promotor 2 & $\mathrm{E}$ & 2600.00 & 500 \\
Promotor 3 & Internet & 200.00 & 50 \\
Promotor 3 & $\mathrm{E}$ & 2600.00 & 500 \\
Multifuncional & $\mathrm{C}$ & 50.00 & 100000 \\
A & $\mathrm{B}$ & 50.00 & 100000 \\
A & $\mathrm{C}$ & 50.00 & 100000 \\
A & $\mathrm{D}$ & 50.00 & 100000 \\
B & $\mathrm{C}$ & 50.00 & 100000 \\
B & $\mathrm{D}$ & 50.00 & 100000 \\
B & $\mathrm{E}$ & 50.00 & 100000 \\
C & $\mathrm{D}$ & 50.00 & 100000 \\
\hline \hline
\end{tabular}

Cuadro 1: Costo y velocidad de enlaces. 


\begin{tabular}{cc|cc}
\hline \hline Nodo A & Nodo B & Req. Mín. de conexión & Clave \\
\hline Créditos & Multifuncional & 1 & 1 \\
Finanzas & Multifuncional & 1 & 1 \\
Contabilidad & Multifuncional & 1 & 1 \\
Dirección & Multifuncional & 1 & 1 \\
Créditos & Servidor 1 & 3 & 2 \\
Finanzas & Servidor 1 & 1 & 1 \\
Contabilidad & Servidor 1 & 1 & 1 \\
Dirección & Servidor 1 & 2 & 2 \\
Créditos & Servidor 2 & 1 & 3 \\
Finanzas & Servidor 2 & 1 & 1 \\
Contabilidad & Servidor 2 & 1 & 1 \\
Dirección & Servidor 2 & 1 & 3 \\
Servidor 1 & Servidor 2 & 1 & 4 \\
Promotor 1 & Servidor 1 & 2 & 5 \\
Promotor 2 & Servidor 1 & 2 & 5 \\
Promotor 3 & Servidor 1 & 2 & 5 \\
Promotor 1 & Servidor 2 & 1 & 5 \\
Promotor 2 & Servidor 2 & 1 & 5 \\
Promotor 3 & Servidor 2 & 1 & 5 \\
Promotor 1 & Créditos & 1 & 5 \\
Promotor 2 & Créditos & 1 & 5 \\
Promotor 3 & Créditos & 1 & 5 \\
\hline \hline
\end{tabular}

Cuadro 2: Requerimientos de conectividad mínima.

- Un grafo no dirigido $G=(V, E)$, siendo $V$ el conjunto de nodos (para este ejemplo, representarían a los equipos de cómputo) y $E$ el conjunto de aristas (que representan a los enlaces de comunicación bidireccionales (full dúplex)).

- Una matriz $C$ de costos no negativos asociados a las aristas del grafo $G$, donde la diagonal principal tiene un valor muy grande simbolizado con una $M$, esto se debe a que no tiene ningún sentido utilizarse así mismo.

- Un subconjunto fijo del conjunto de nodos, $T \subseteq V$ llamado conjunto de nodos terminales, de cardinalidad $\|T\|=n_{T}$, tal que $2 \leq n_{T} \leq n$, siendo $n$ la cardinalidad del conjunto de nodos $V$, es decir $\|V\|=n$.

- Una matriz simétrica $R=r_{i j}$ con $i, j \in T$, de dimensión $n_{T} \times n_{T}$, 
cuyos elementos son enteros no negativos que indican los requerimientos de conectividad -cantidad de caminos disjuntos requeridosentre todo par de nodos terminales.

El problema Steiner generalizado propone encontrar $G_{T}$, un subgrafo de $G$ de costo mínimo, que sea un grafo de cubrimiento del conjunto de nodos terminales. Todo par de nodos terminales $i, j \in T, i \neq j$ deberán ser localmente $r_{i j}$ arista-conexos en $G_{T}$. Esto significa que deben existir $r_{i j}$ caminos disjuntos, que no comparten aristas entre los nodos terminales $i$ y $j$ en $G_{T}$. Sobre los nodos no pertenecientes al conjunto de nodos terminales no se plantean requisitos de conectividad. Estos nodos, conocidos como nodos de Steiner, pueden formar parte o no de la solución óptima, dependiendo de la conveniencia de utilizarlos. La descripción previa corresponde a la versión arista-conexa del problema Steiner generalizado, utilizada para modelar problemas sobre redes en las cuales se supone a los enlaces (aristas) sujetos a fallas, pero los nodos se suponen perfectos(aunque en la realidad no necesariamente es cierto).

Considérese el grafo $G$ de la Figura 2, donde los nodos terminales se presentan en color oscuro, mientras que los nodos de Steiner se presentan con color claro. Los costos asociados a cada arista son una ponderación entre el costo de la arista y la velocidad de la conexión presentados en el Cuadro 1, mientras que los requerimientos de conectividad corresponden al Cuadro 2. El costo total de un camino dado entre dos nodos está dado por medio de un cociente entre el costo monetario y la velocidad de conexión, la relación propuesta es la siguiente:

$$
C c=\frac{\sum_{i=0}^{n} \sum_{j=0}^{n}\left(c_{i j}\right) v_{i j}}{\min _{i, j=0 \rightarrow n}\left\{v_{i j} x_{i j} \| v_{i j} x_{i j}>0\right\}}
$$

donde:

- $C c$ es el costo del camino,

- $c_{i j}$ es el costo de la arista $\mathrm{i}, \mathrm{j}$,

- $v_{i j}$ es la velocidad de transmisión de la arista ij,

- $x_{i j}= \begin{cases}1 & \text { para cuando la arista } i j \text { está en el camino, } \\ 0 & \text { en otro caso }\end{cases}$ 


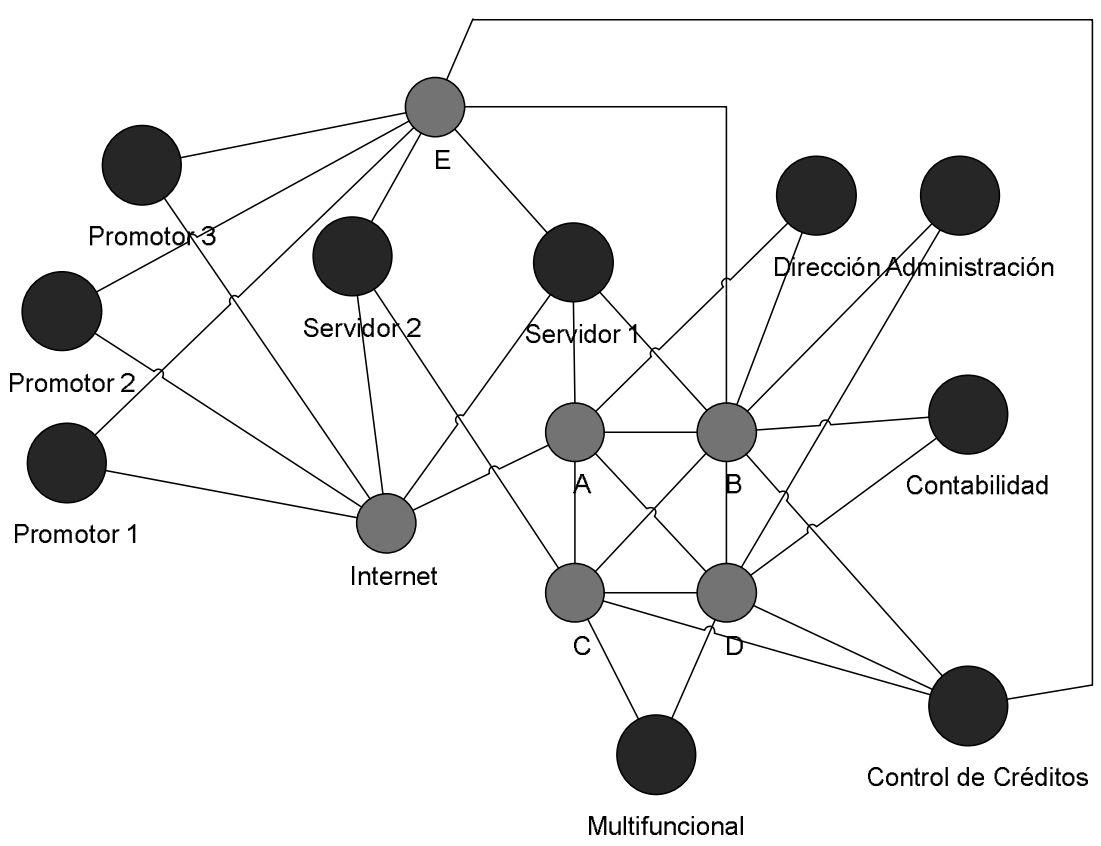

Figura 2: Gráfica del ejemplo como problema tipo Steiner.

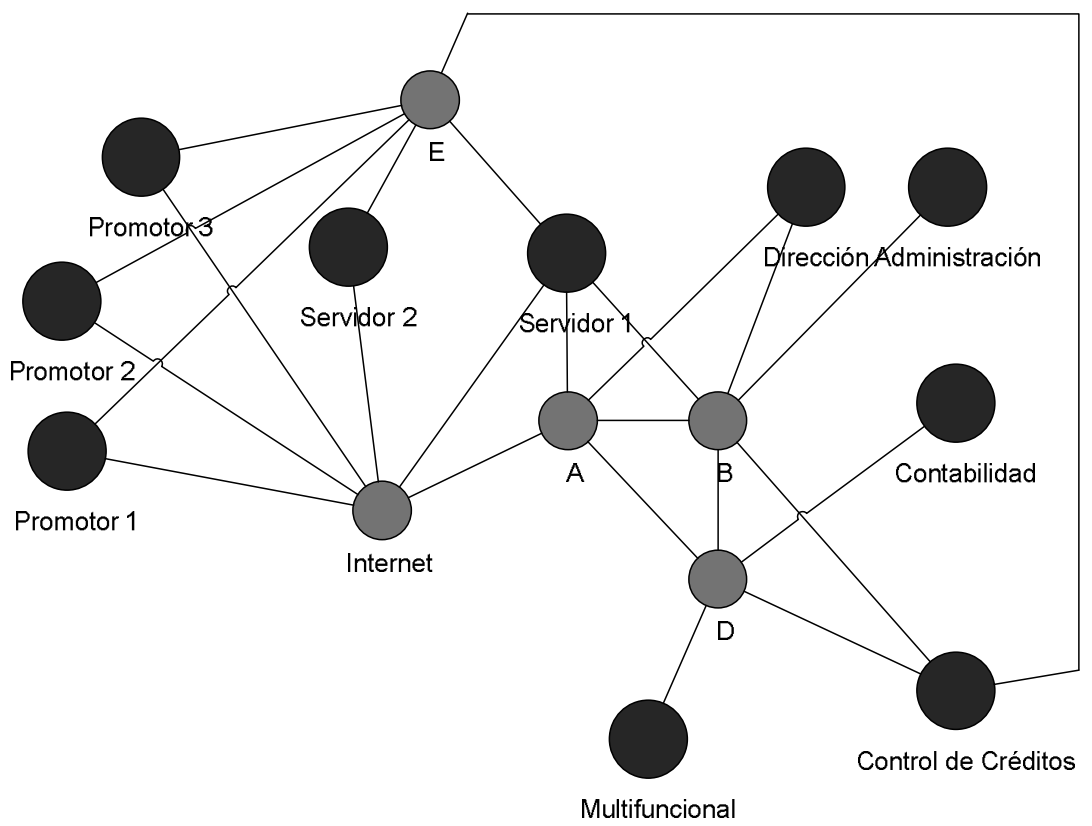

Figura 3: Gáfica de la solución del ejemplo. 


\begin{tabular}{|c|c|c|}
\hline$\overline{\text { Nodo } 1}$ & $\overline{\text { Nodo } 2}$ & Caminos $\{\ldots\}$ \\
\hline Créditos & Multifuncional & $\{$ Créditos, D, Multifuncional $\}$ \\
\hline Administración & Multifuncional & $\{$ Administración, B, D, Multifuncional $\}$ \\
\hline Contabilidad & Multifuncional & $\{$ Contabilidad, B, D, Multifuncional $\}$ \\
\hline Dirección & Multifuncional & $\{$ Dirección, A, D, Multifuncional $\}$ \\
\hline Créditos & Servidor1 & $\begin{array}{c}\text { \{Créditos, D, A, Servidor } 1\}, \\
\text { \{Créditos, B, Servidor } 1\} \\
\text { \{Créditos, E, Servidor } 1\}\end{array}$ \\
\hline Contabilidad & Servidor 1 & $\{$ Créditos, B, Servidor 1$\}$ \\
\hline Dirección & Servidor 1 & $\begin{array}{l}\{\text { Dirección, A, Servidor } 1\}, \\
\{\text { Dirección, B, Servidor } 1\} .\end{array}$ \\
\hline Administración & Servidor 1 & $\{$ Administración, B, Servidor1\} \\
\hline Créditos & Servidor 2 & $\{$ Créditos, E, Servidor 2$\}$ \\
\hline Contabilidad & Servidor 2 & $\begin{array}{l}\text { \{Contabilidad, B, A, } \\
\text { Internet, Servidor } 2\}\end{array}$ \\
\hline Dirección & Servidor 2 & $\{$ Dirección, A, Internet, Servidor 2$\}$ \\
\hline Administración & Servidor 2 & $\begin{array}{l}\text { \{Administración, B, A, } \\
\text { Internet, Servidor } 2\}\end{array}$ \\
\hline Promotores & Servidor 1 & $\begin{array}{c}\{\text { Promotores, Internet, Servidor } 1\} \\
\{\text { Promotores, E, Servidor } 1\}\end{array}$ \\
\hline Promotores & Servidor2 & $\{$ Promotores, E, Servidor 2$\}$ \\
\hline Promotores & Créditos & \{Promotores, E, Créditos\} \\
\hline
\end{tabular}

Cuadro 3: Caminos solución al problema.

\section{Resultados y conclusiones}

Como consecuencia de la complejidad de la clase de problemas Steiner, su resolución utilizando algoritmos exactos se hace cada vez menos tratable al aumentar el tamaño de los problemas. Por tal motivo, se buscan soluciones alternativas utilizando heurísticas que permitan encontrar soluciones de calidad aceptable en tiempos razonables.

Una variada gama de heurísticas específicas y algoritmos de aproximación se han propuesto para la resolución de la clase de problemas Steiner, en especial para el problema del árbol de Steiner y los problemas de $k$ conexión. A manera de ejemplo, es posible citar las heurísticas específicas más populares que se han desarrollado para la resolución del problema del árbol de Steiner: Minimum Cost Paths (MCP) propuesta por Takahashi y Matsuyama(1980), Distance Network Heuristic (DNH) propuesta por Kou 
et al.(1981), Average Distance Heuristic (ADH) propuesta por RaywardSmith(1983) y revisada por Rayward-Smith y Clare(1986).

Respecto al Problema Steiner Generalizado, es posible indicar que no se ha estudiado en su formulación más genérica del mismo modo que ha sido estudiado el problema del árbol de Steiner y, hasta cierto nivel, los problemas de $k$ conexión. En general los investigadores han concentrado sus esfuerzos en resolver casos particulares o simplificados.

La Figura 3 presenta una solución de costo mínimo para la instancia del problema Steiner generalizado, se utilizó un algoritmo glotón planteado sobre el grafo $G$ de la Figura 2, sin embargo se puede ampliar a heurísticos que nos proporcionen mejores resultados si es que los hay. El costo mínimo mencionado considera el costo del grafo completo. Para conectar los 10 nodos terminales y verificar los requerimientos de conectividad, la solución incluye 5 de los 6 nodos de Steiner originales, seleccionados como los óptimos en términos del número y posición considerando los costos de los enlaces correspondientes. Asimismo, el grafo solución incluye 22 de las 31 aristas del grafo original, disminuyendo el costo total monetario de un costo original de $21,350 u$.m. mensual a un costo de $15,950 u$.m. mensual en este diseño mejor(no necesariamente el óptimo), lo que implica en un ahorro de más del $32 \%$ por mes, evitando el gasto de $64,800 u . m$. al año. Tomando en cuenta que la velocidad mínima del grafo solución es de 2,000 kbps obtenemos un costo de 7.98 u.m./kbps usando el cociente precio/velocidad, en comparación con un valor de 427 u.m./kbps del grafo original cuya velocidad mínima es de $50 \mathrm{kbps}$. Al tomar en cuenta la velocidad y no únicamente las unidades monetarias aseguramos que en situaciones en las que dos enlaces con velocidades diferentes y precios parecidos se eligira la que tenga mejor relación precio/velocidad y no solamente la que tenga el menor costo monetario. La solución presentada no necesariamente es única, ya que otras soluciones óptimas pueden existir para el problema. La solución de costo mínimo presentada cumple con los requerimientos mínimos de conectividad propuestos en la Tabla 2. Los caminos disjuntos para cada requerimiento pueden observarse en el Cuadro 3.

\section{Agradecimientos}

Los autores desean agradecer los valiosos comentarios de los árbitros anónimos, lo que contribuó a una mejora del presente trabajo. 


\section{Referencias}

[1] Crescenzi, P.; Kann, V. (2005) "A compendium of NP optimization problems". Retrieved Junio 8, 2006 from KTH NADA: http://www.nada.kth.se/ viggo/problemlist/compendium.html.

[2] Faloutsos, M. (2004) "The Steiner's tree problem". Retrieved Junio 9, 2006 from Department of Computer Science and Engineering at the University of California, Riverside: http://www.cs.ucr.edu/ michalis/COURSES/240-04/steiner.html.

[3] Kou, L.; Markowsky, G.; Berman, L. (1981) "A fast algorithm for Steiner trees", Acta Inf. 15: 141-145.

[4] Nesmachnow, S. (2004) "Algoritmos genéticos paralelos y su aplicación al diseño de redes de comunicación confiables", PEDECIBA, Montevideo, Uruguay.

[5] Nesmachnow, S.; Cancela, H.; Enrique, A. (2004) "Técnicas evolutivas aplicadas al diseño de redes de comunicaciones confiables", Tercer Congreso Español de Metaheurísticas, Algoritmos Evolutivos y Bioinspirados, Córdoba: 388-395.

[6] Rayward-Smith, V.J. (1983) "The computation of nearly minimal steiner trees in graphs", Mathematics, Education, Science, Technology 14: 15-23.

[7] Rayward-Smith, V.J.; Clare, A. (1986) "On finding Steiner vertices", Networks 16: 283-294.

[8] Tanenbaun, A. (1997) "Algoritmos de enrutamiento", in: A. Tanenbaun, C. Trentacoste \& N. Regina (Eds.) Redes de Computadoras, Prentice-Hall, Amsterdam: 345-365.

[9] Takahashi, H.; Matsuyama ,A. (1980) "An approximate solution for the Steiner problem in graphs", Mathematica Japonica 24: 573-577. 